\title{
Usefulness of Consciousness Sedation with Dexmedetomidine and Pentazocine during Endovascular Treatment for Acute Stroke
}

\author{
Kouhei Nir, ${ }^{1}$ Hayatsura HanAdA, ${ }^{1}$ Fumihiro HIRAOKA, ${ }^{1}$ Ayumu ETO, ${ }^{1}$ \\ Takafumi MiTSUTAKE, ${ }^{1}$ and Masanori TSUTSUMI ${ }^{1}$ \\ ${ }^{1}$ Department of Neurosurgery, Fukuoka University Chikushi Hospital, \\ Chikushino, Fukuoka, Japan
}

\begin{abstract}
We investigated the safety and efficacy of consciousness sedation with dexmedetomidine (DEX) during the endovascular treatment of patients with acute cerebral infarction. Between April 2014 and November 2016, 38 stroke patients underwent endovascular thrombectomy (EVT) under local anesthesia and DEX consciousness sedation. The continuous intravenous administration of low-dose DEX $(0.3-0.4 \mu \mathrm{g} / \mathrm{kg} / \mathrm{h}) \mathrm{was}$ started before entering the operating room. Patients not completely immobilized received an iv bolus of pentazocine (PTZ) and/or DEX (0.5-0.6 $\mu \mathrm{g} / \mathrm{kg} / \mathrm{h})$. EVT was performed using a stent retriever and/or direct thrombo-aspiration. DEX sedation was stopped as soon as the operation was finished. A stent retriever was used in $8(21.1 \%)$, direct thrombo-aspiration in $10(26.3 \%)$, a stent retriever plus thrombo-aspiration in $14(36.8 \%)$, and other devices in 6 patients $(15.8 \%)$. Reperfusion exceeding $50 \%$ (thrombolysis in cerebral infarction $>2$ b) was obtained in 30 patients $(78.9 \%)$. DEX sedation alone immobilized 24 patients $(63.2 \%), 14(36.8 \%)$ required the additional injection of DEX or PTZ when EVT devices were navigated to the lesion. The administration of DEX and PTZ elicited no significant respiratory depression or cardiac dysfunction interfering with the procedures. Consciousness sedation by DEX was effective during the endovascular treatment of acute stroke patients. Under DEX sedation and the injection of PTZ, EVT was possible without significantly changing the patients' vital signs.
\end{abstract}

Key words: acute cerebral infarction, endovascular thrombectomy, immobilization local anesthesia, consciousness sedation, dexmedetomidine

\section{Introduction}

Advances in the development of devices and pharmaceuticals render the endovascular treatment of patients with acute ischemic stroke effective and safe. ${ }^{1)}$ However, treatment outcomes depend on the patient age, reperfusion grade, time to reperfusion, and the site of vessel occlusion., ${ }^{2,3)}$ The proper anesthetic, local- or general anesthesia, to be used in patients requiring prompt endovascular treatment remains controversial. Local anesthesia requires optimal consciousness sedation for immobilization, anxiety alleviation, and pain control. However,

Received September 26, 2017; Accepted October 16, 2017

Copyright@ 2018 by The Japan Neurosurgical Society This work is licensed under a Creative Commons AttributionNonCommercial-NoDerivatives International License. excessive consciousness sedation with, for example, diazepam, benzodiazepine, remifentanil, or propofol, may elicit respiratory depression and blood pressure fluctuation, while inadequate sedation fails to immobilize the patient and may require subsequent general anesthesia and intubation. ${ }^{4-6)}$

We investigated the feasibility of local anesthesia using dexmedetomidine (DEX) and pentazocine (PTZ) during the endovascular treatment of patients with acute cerebral infarction.

\section{Materials and Methods}

Our single-center registry showed that between April 2014 and November 2016, 40 patients with acute cerebral infarction underwent endovascular treatment. Of these, 38 were treated under DEX sedation. The ethics committee of our institution approved this study and informed consent was obtained from 
all patients or their legal representatives after the procedures had been fully explained.

The low-dose intravenous (iv) injection of DEX $(0.3-0.4 \mathrm{\mu g} / \mathrm{kg} / \mathrm{h})$ was started before entering the operating room. Patients not completely immobilized or reporting pain from operative manipulations received an iv bolus injection of PTZ $(0.3 \mathrm{mg} /$ $\mathrm{kg}$ ) and/or additional DEX $(0.5-0.6 \mu \mathrm{g} / \mathrm{kg} / \mathrm{h})$. The continuous infusion of DEX was stopped as soon as the operation was finished even in patients with incomplete reperfusion (Fig. 1).

All patients underwent endovascular thrombectomy (EVT) using a stent retriever and/or direct thromboaspiration. In patients with arterial occlusion in the anterior circulation, (i.e., the internal carotid artery [ICA], middle cerebral artery [MCA], or anterior cerebral artery [ACA]), a 9-French guiding balloon catheter was navigated to the ICA via the femoral approach, then a microcatheter (Marksman; Medtronic, Minneapolis, MN, USA) was passed through the occlusion site using a microguidewire via a Penumbra 5MAX ACE device (Penumbra Inc., Alameda, CA, USA). A 4 to 6-mm diameter Solitaire FR (Medtronic) was used for EVT; it was retrieved during thrombo-aspiration with a Penumbra 5MAX ACE device after temporary blocking of the ICA flow. In patients with arterial occlusion in the posterior circulation, (i.e., the vertebral artery [VA],

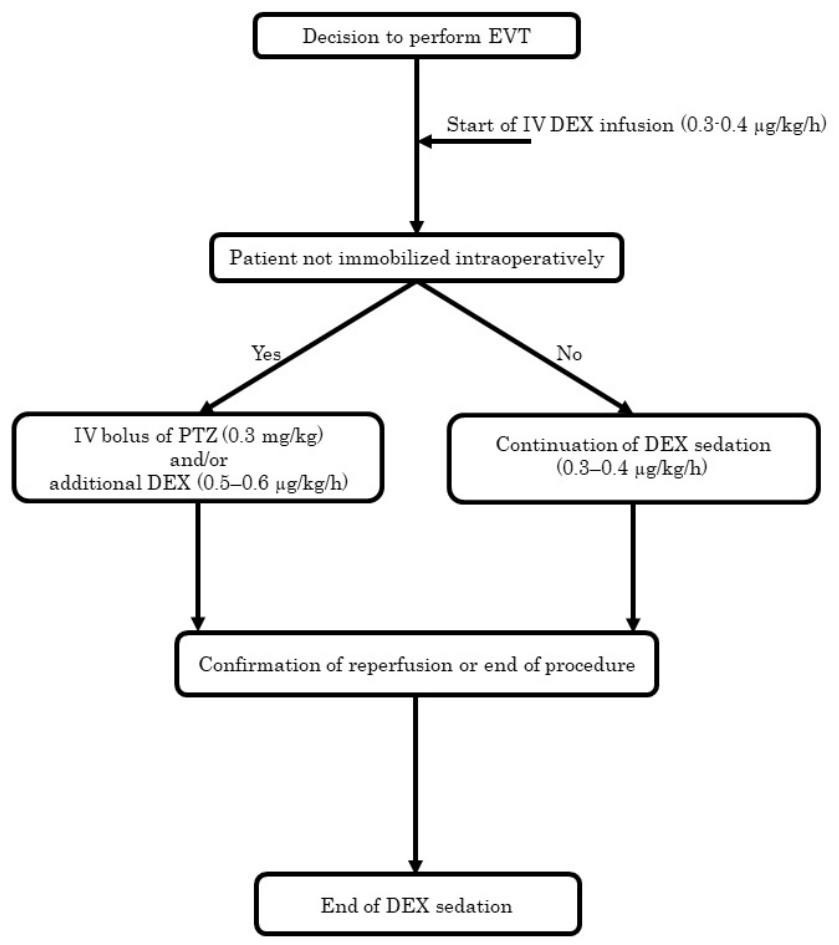

Fig. 1 Flowchart of dexmedetomidine and pentazocine administration for conscious sedation during endovascular thrombectomy. EVT: endovascular thrombectomy, IV: intravenous, DEX: dexmedetomidine, PTZ: pentazocine. basilar artery [BA], or posterior cerebral artery [PCA]), a 6-French guiding catheter was navigated to the VA and direct thrombo-aspiration with a Penumbra 3MAX device or a stent retriever and a 4-mm diameter Solitaire FR device was used for EVT. Other devices such as a balloon catheter were selected when occlusion was not resolved by EVT. The grade of posttreatment revascularization was evaluated by assigning a thrombolysis in cerebral infarction (TICI) grade. ${ }^{7)}$

All patients underwent diffusion-weighted magnetic resonance imaging (DW-MRI) before and after EVT to check for new ischemic lesions. The DW Alberta Stroke Program Early CT Score (DW-ASPECTS) was used to evaluate the size of the ischemic area. Pre- and post-procedural neurologic findings were evaluated on the National Institute of Health Stroke Scale (NIHSS) and the modified Rankin Scale (mRS), respectively. Vital- and neurologic signs were monitored throughout the operation. The systolic blood pressure (SBP) and pulse rate (PR) were measured at 5-min intervals with an automated sphygmomanometer placed on the patient's upper arm. Abnormal SBP was defined as $<80 \mathrm{mmHg}$ or $>180 \mathrm{mmHg}$, abnormal PR as $<60$ beats/min or $>100$ beats $/ \mathrm{min}$. Respiratory depression and cardiac dysfunction that could interfere with the procedure were recorded. Symptomatic intracranial hemorrhage (SICH) was recorded when the NIHSS worsened by more than 4 points.

Continuous variables were expressed as the mean \pm 1 standard deviation, categorical variables as percentages. Our clinical outcomes were compared to previous randomized studies. The Fisher exact test was used for categorical- and the Mann-Whitney $U$-test for continuous variables. A $P$ value $<0.05$ was considered statistically significant.

\section{Results}

The sample group included 20 men $(52.6 \%)$ and 18 women $(47.4 \%)$ with a mean age of $73.5 \pm 10.7$ years. The preoperative mean NIHSS was $16 \pm 7.4$, the mean DW-ASPECTS was $7.0 \pm 2.1$. Of the 38 arterial occlusions, $16(42.1 \%)$ were in the ICA, 18 (47.4\%) were in the MCA, the others were in the VA, PCA, or BA (Table 1).

Angiographic and operative findings are also shown in Table 1. Before EVT, 20 patients (52.6\%) were injected with recombinant tissue plasminogen activator (rt-PA). A stent retriever alone was used to address $8(21.1 \%)$, direct thrombo-aspiration alone in $10(26.3 \%)$, a stent retriever plus thromboaspiration in $14(36.8 \%)$, and other devices in 6 lesions (15.8\%). Reperfusion exceeding 50\% (TICI $>2$ b) was obtained after 30 EVTs $(78.9 \%$ ). 
Table 1 Characteristics and operative findings in 38 stroke patients treated by endovascular thrombectomy under local anesthesia

\begin{tabular}{|c|c|}
\hline Patient characteristics & All patients $(n=38)$ \\
\hline Age (mean \pm SD, y) & $73.5 \pm 10.7$ \\
\hline Male, $n(\%)$ & $20(52.6)$ \\
\hline Female, $n(\%)$ & $18(47.4)$ \\
\hline NIHSS (mean \pm SD) & $16 \pm 7.4$ \\
\hline DW-ASPECTS (mean \pm SD) & $7 \pm 2.1$ \\
\hline \multicolumn{2}{|l|}{ Occlusion location } \\
\hline ICA, $n(\%)$ & $16(42.1)$ \\
\hline MCA, $n(\%)$ & $18(47.4)$ \\
\hline VA, $n(\%)$ & $1(2.6)$ \\
\hline $\mathrm{BA}, n(\%)$ & $2(5.3)$ \\
\hline PCA, $n(\%)$ & $1(2.6)$ \\
\hline Intravenous rt-PA, $n(\%)$ & $20(52.6)$ \\
\hline \multicolumn{2}{|l|}{ EVT devices } \\
\hline SR, $n(\%)$ & $8(21.1)$ \\
\hline ADAPT, $n(\%)$ & $10(26.3)$ \\
\hline SR + ADAPT, $n(\%)$ & $14(36.8)$ \\
\hline Other devices, $n(\%)$ & $6(15.8)$ \\
\hline Reperfusion (TICI > 2b), $n(\%)$ & $30(78.9)$ \\
\hline \multicolumn{2}{|l|}{ Sedation, $n(\%)$} \\
\hline DEX, $n(\%)$ & $24(63.2)$ \\
\hline DEX + PTZ, $n(\%)$ & $14(36.8)$ \\
\hline Respiratory depression, $n(\%)$ & 0 \\
\hline Cardiac dysfunction, $n(\%)$ & 0 \\
\hline Pre SBP (mean \pm SD mmHg) & $144.5 \pm 20.9$ \\
\hline Post SBP (mean \pm SD mmHg) & $137.6 \pm 18.9$ \\
\hline
\end{tabular}

ADAPT: a direct aspiration first-pass technique, BA: basilar artery, DEX: dexmedetomidine, DW-ASPECTS: diffusionweighted magnetic resonance imaging Alberta Stroke Program Early CT Score, EVT: endovascular thrombectomy, ICA: internal carotid artery, $n$ : number of patients, MCA: middle cerebral artery, NIHSS: National Institutes of Health Stroke Scale, PCA: posterior cerebral artery, PTZ: pentazocine, rt-PA: recombinant tissue plasminogen activator, SBP: systolic blood pressure, SD: standard deviation, SR: stent retriever, TICI: Thrombolysis in Cerebral Infarction, VA: vertebral artery.
DEX and PTZ were effective in all patients; 24 $(63.2 \%)$ were immobilized by DEX alone, 14 (36.8\%) required the additional administration of DEX and PTZ when EVT devices were navigated to the lesion. Patients without severe preoperative neurologic findings were easily awakened by voice after stopping DEX sedation which elicited no significant respiratory depression or cardiac dysfunction interfering with the procedure. The mean pre- and post-SBP was 144.5 $\pm 20.9 \mathrm{mmHg}$ and $137.6 \pm 18.9 \mathrm{mmHg}$, respectively, and not significantly different $(P=0.11)$.

Table 2 shows the EVT devices used and the sedation and analgesic (PTZ) drugs administered in the 38 cases. DEX plus PTZ was used in 6 stent retriever cases, 2 cases of direct thrombo-aspiration, 5 cases of stent retriever plus thrombo-aspiration, and 1 case using other devices. PTZ was needed in 11 of $22(50 \%)$ cases using stent retriever with or without thrombo-aspiration and in 3 of 16 (18.8\%) cases using direct thrombo-aspiration alone or other devices. The higher rate of PTZ usage during stent retriever manipulations was noted, however, there was no statistically significant difference in PTZ usage based on the type of EVT device used $(P=0.09)$.

Table 3 compares our procedural and clinical outcomes with those reported by others. ${ }^{4,6)}$ In this study, the mRS 30 days after stroke onset was 0-2 in $14(36.8 \%)$ and $3-5$ in $16(42.1 \%)$ patients. Of the 38 patients $8(21.1 \%)$ died due to progressive infarction without reperfusion $(n=5)$, of pneumonia $(n=2)$, or of pancreatic cancer $(n=1)$; 3 patients $(7.9 \%)$ developed SICH.

Our good reperfusion rate (TICI $>2$ b) was significantly higher than that reported by van den Berg et al. $\left.{ }^{6}\right)(P<0.001)$ and none of our patients required a switch to general anesthesia. The rate of a good clinical outcome (mRS 0-2), mortality, and symptomatic intracranial hemorrhage was not different from that reported by van den Berg et al. ${ }^{6)}$ (mRS 0-2: $P>0.17$, mortality: $P>0.49$, symptomatic intracranial hemorrhage: $P>0.59$ ).

We also compared our- with findings reported by Schönenberger et al. ${ }^{4}$ who placed their patients

Table 2 Relationship between devices/techniques and sedation/analgesic drugs used for endovascular thrombectomy

\begin{tabular}{lccc}
\hline \multicolumn{2}{l}{ All patients $(n=38)$} & $\mathrm{DEX}(n=24,63.2 \%)$ & $\mathrm{DEX}+\mathrm{PTZ}(n=14,36.8 \%)$ \\
\hline EVT device/technique & $8(21.1)$ & $2(8.3)$ & $6(42.9)$ \\
SR, $n(\%)$ & $10(26.3)$ & $8(33.3)$ & $2(14.3)$ \\
ADAPT, $n(\%)$ & $14(36.8)$ & $9(37.5)$ & $5(35.7)$ \\
SR + ADAPT, $n(\%)$ & $6(15.8)$ & $5(20.8)$ & $1(7.1)$ \\
Other devices, $n(\%)$ & & \\
\hline
\end{tabular}

ADAPT: a direct aspiration first-pass technique, DEX: dexmedetomidine, EVT: endovascular thrombectomy, n: number of patients, PTZ: pentazocine, SR: stent retriever. 
Table 3 Comparison of the procedures and outcomes in our- and earlier studies

\begin{tabular}{lccc}
\hline & Our study $(n=38)$ & Ref. 6 CS group $(n=278)$ & Ref. 4 GA group $(n=73)$ \\
\hline Reperfusion (TICI $>2$ b), $n(\%)$ & $30(78.9)$ & $113(40.6)$ & $65(89.0)$ \\
Conversion to GA, $n(\%)$ & 0 & $10(3.6)$ & NA \\
mRS 0-2, $n(\%)$ & $14(36.8)$ & $72(25.9)$ & $27(37.0)$ \\
Mortality, $n(\%)$ & $8(21.1)$ & $46(16.5)$ & $18(24.7)$ \\
SICH, $n(\%)$ & $3(7.9)$ & $33(11.9)$ & Not provided \\
\hline
\end{tabular}

CS: consciousness sedation, GA: general anesthesia, mRS: modified Rankin scale, n: number of patients, NA: not applicable, SICH: symptomatic intracranial hemorrhage, TICI: thrombolysis in cerebral infarction.

under general anesthesia. Again, the rate of good reperfusion, a good clinical outcome, and mortality was not significantly different from theirs (mRS 0-2: $P=1.0$, mortality: $P=0.81$ ).

\section{Discussion}

According to Brinjikji et al., ${ }^{8)}$ EVT under consciousness sedation may improve the outcome in patients with acute ischemic stroke. The reduction in the cerebral blood flow to the ischemic penumbra was milder under consciousness sedation than under general anesthesia which typically elicits hypotension. ${ }^{9)}$ Davis et al. ${ }^{10)}$ reported that patients whose SBP was lower than $140 \mathrm{mmHg}$ tended to have undergone EVT under general anesthesia.

Others ${ }^{4,5)}$ found that treatment under general anesthesia was effective and did not elicit hypotension. Wang et al. ${ }^{11)}$ reported that general anesthesia during emergency endovascular stroke treatment had no deleterious effect on the mRS-based outcome. At their institution, the anesthesia team was in the hospital and available $24 \mathrm{hrs}$ per day; therefore, there was minimal delay with the use of general anesthesia. In most Japanese hospitals, however, the anesthesia team is not present in the hospital at all times. Moreover, EVTs may be performed by one operator alone. While many interventionalists understand the benefits of performing EVTs under general anesthesia, this option is not available for all stroke patients.

As intraoperative neurological assessment is possible in patients under consciousness sedation, such real-time evaluations may reduce complications due to EVT procedures. ${ }^{2,12)}$ However, the time required for EVT may be prolonged in uncooperative awake patients because motion artifacts may require repeat angiography. According to Abou-Chebl et al.,2) in patients under consciousness sedation, the time required for fluoroscopy is also extended. Additional sedation, required to maintain immobilization in uncooperative awake patients, may result in respiratory depression and a change from conscious sedation to general anesthesia was needed in patients with respiratory depression, coma, or the loss of airway-protective reflexes. ${ }^{10,13)}$ Moreover, high-dose sedatives may also prolong consciousness disturbance and hamper the decision of whether to perform further procedures.

Huncke et al. ${ }^{14)}$ reported the efficacy of DEX sedation during vascular procedures and Whalin et al. ${ }^{15}$ demonstrated that DEX can be safely administered in patients undergoing endovascular reperfusion therapy. DEX, a centrally acting $\alpha-2$ receptor agonist, was originally introduced as a sedative for critically ill mechanically ventilated patients. Patients are easily aroused with verbal stimuli and DEX sedation does not appear to be associated with significant respiratory depression because DEX sedation mimics natural sleep. ${ }^{16,17)}$ In addition, DEX is a rapidly-metabolized drug with a plasma half-time of approximately 2 h. ${ }^{18)}$ Except for the non-reperfused patients, our patients were easily aroused by voice during the operation, and they awoke without delay after stopping DEX sedation.

Chi et al. ${ }^{19)}$ suggested that DEX-induced vasoconstriction in the normal cortex helps to redistribute the blood flow to the ischemic reperfused cortex, thereby contributing to a more complete restoration of the blood flow to this region. However, Nakano and Okamoto ${ }^{20)}$ reported that high-dose DEX resulted in hypertension and exacerbated ischemic brain injury. On the other hand, DEX may result in a dosedependent decrease in the pulse rate and blood pressure, and the initial DEX bolus may elicit a biphasic blood pressure change. ${ }^{14)}$ Therefore, we administered low-dose DEX during the procedure. As our rate of good clinical outcomes and SICH was comparable to earlier randomized studies, ${ }^{4,6)}$ DEX sedation did not adversely affect the clinical outcomes in our patients.

Pain during the manipulation of EVT devices may result in intraoperative motion artifacts. The sedative effect of DEX may be attributable to $\alpha 2$ adrenergic receptor-dependent downstream mechanisms. ${ }^{21)}$ In our series, 24 of 38 patients were sufficiently immobilized by DEX sedation alone, 11 others subjected to the direct thrombo-aspiration and/or the use of a stent retriever required the additional administration of PTZ. 
In Japan, PTZ, a kappa opioid receptor agonist, is frequently used as a non-morphine analgesic during minimally invasive surgery. We added it to DEX in our uncooperative patients and obtained stable consciousness sedation and pain amelioration. Although there was no statistically significant difference in the rate of PTZ administration among the EVT device types used, we suspect that the manipulation of the stent retriever is more painful than of other devices.

Analgesics induce central respiratory depression in a dose-dependent manner and the combination of sedatives and analgesics may exacerbate respiratory depression. Our sedation/pain relief regimen consisted of a low-dose combination; the addition of PTZ to DEX yielded sufficient consciousness sedation and pain relief. We posit that the onset of respiratory depression was inhibited because the administration of low-dose DEX resulted in adequate consciousness sedation.

Our study has some limitations. The choice of EVT devices depended on the site of the arterial occlusion and the preference of the surgeon. Patient immobilization was influenced by the patient's consciousness level or motor disturbance reflected in the preoperative NIHSS. Also, we did not have 90-day outcome data available for analysis, which limits our ability to evaluate long-term outcomes. However, as rehabilitation often improves neurological assessment, we would expect our rate of good clinical outcome to increase at 90 days. In the current study, we focused on the effect of different device types and techniques and of preoperative neurologic findings on motion artifacts elicited during the EVT procedure. Studies are underway to elucidate the effect of our regimen for consciousness sedation in larger patient populations and to compare the clinical outcomes in patients treated with EVT under different types of consciousness sedation and under general anesthesia.

\section{Conclusion}

Local anesthesia using DEX sedation was effective during the endovascular treatment of patients with acute stroke. In some patients, low-dose DEX alone failed to adequately control pain during device manipulation, however, the addition of PTZ allowed completion of the EVT procedure without significant respiratory depression or hemodynamic instability.

\section{Acknowledgment}

We are grateful to Kimiya Sakamoto, Ritsuro Inoue, Yusuke Morinaga, and Misato Kawaguchi for their help with this study.

\section{Conflicts of Interest Disclosure}

The authors have no personal financial or institutional interests in any of the drugs, materials, or devices used in this study. All authors are members of the Japan Neurosurgical Society and all have registered self-reported COI Disclosure Statement Forms on-line.

\section{References}

1) Berkhemer OA, Fransen PS, Beumer D, et al.: A randomized trial of intraarterial treatment for acute ischemic stroke. N Engl J Med 372: 11-20, 2015

2) Abou-Chebl A, Lin R, Hussain MS, et al.: Conscious sedation versus general anesthesia during endovascular therapy for acute anterior circulation stroke: preliminary results from a retrospective, multicenter study. Stroke 41: 1175-1179, 2010

3) Khatri P, Abruzzo T, Yeatts SD, Nichols C, Broderick JP, Tomsick TA: Good clinical outcome after ischemic stroke with successful revascularization is time-dependent. Neurology 73: 1066-1072, 2009

4) Schönenberger S, Uhlmann L, Hacke W, et al.: Effect of conscious sedation vs general anesthesia on early neurological improvement among patients with ischemic stroke undergoing endovascular thrombectomy: a randomized clinical trial. JAMA 316: 1986-1996, 2016

5) Slezak A, Kurmann R, Oppliger L, et al.: Impact of anesthesia on the outcome of acute ischemic stroke after endovascular treatment with the solitaire stent retriever. AJNR Am J Neuroradiol 38: 1362-1367, 2017

6) van den Berg LA, Koelman DL, Berkhemer OA, et al.: Type of anesthesia and differences in clinical outcome after intra-arterial treatment for ischemic stroke. Stroke 46: 1257-1262, 2015

7) Noser EA, Shaltoni HM, Hall CE, et al.: Aggressive mechanical clot disruption: a safe adjunct to thrombolytic therapy in acute stroke? Stroke 36: 292-296, 2005

8) Brinjikji W, Murad MH, Rabinstein AA, Cloft HJ, Lanzino G, Kallmes DF: Conscious sedation versus general anesthesia during endovascular acute ischemic stroke treatment: a systematic review and metaanalysis. AJNR Am J Neuroradiol 36: 525-529, 2015

9) Stead LG, Gilmore RM, Vedula KC, Weaver AL, Decker WW, Brown RD: Impact of acute blood pressure variability on ischemic stroke outcome. Neurology 66: 1878-1881, 2006

10) Davis MJ, Menon BK, Baghirzada LB, et al.: Anesthetic management and outcome in patients during endovascular therapy for acute stroke. Anesthesiology 116: 396-405, 2012

11) Wang A, Stellfox M, Moy F, et al.: General anesthesia during endovascular stroke therapy does not negatively impact outcome. World Neurosurg 99: 638-643, 2017 
12) Gupta R: Local is better than general anesthesia during endovascular acute stroke interventions. Stroke 41: 2718-2719, 2010

13) Li F, Deshaies EM, Singla A, et al.: Impact of anesthesia on mortality during endovascular clot removal for acute ischemic stroke. J Neurosurg Anesthesiol 26: 286-290, 2014

14) Huncke TK, Adelman M, Jacobowitz G, Maldonado T, Bekker A: A prospective, randomized, placebocontrolled study evaluating the efficacy of dexmedetomidine for sedation during vascular procedures. Vasc Endovascular Surg 44: 257-261, 2010

15) Whalin MK, Lopian S, Wyatt K, et al.: Dexmedetomidine: a safe alternative to general anesthesia for endovascular stroke treatment. J Neurointerv Surg 6: $270-275,2014$

16) Ebert TJ, Hall JE, Barney JA, Uhrich TD, Colinco MD: The effects of increasing plasma concentrations of dexmedetomidine in humans. Anesthesiology 93: 382-394, 2000

17) Hall JE, Uhrich TD, Barney JA, Arain SR, Ebert TJ: Sedative, amnestic, and analgesic properties of small-dose dexmedetomidine infusions. Anesth Analg 90: 699-705, 2000

18) Movafegh A, Shoeibi G, Ansari M, Sadeghi M, Azimaraghi O, Aghajani Y: Naloxone infusion and post-hysterectomy morphine consumption: a doubleblind, placebo-controlled study. Acta Anaesthesiol Scand 56: 1241-1249, 2012

19) Chi OZ, Grayson J, Barsoum S, Liu X, Dinani A, Weiss HR: Effects of dexmedetomidine on microregional $\mathrm{O} 2$ balance during reperfusion after focal cerebral ischemia. J Stroke Cerebrovasc Dis 24: 163-170, 2015

20) Nakano T, Okamoto H: Dexmedetomidine-induced cerebral hypoperfusion exacerbates ischemic brain injury in rats. J Anesth 23: 378-384, 2009

21) Fan W, Yang H, Sun $Y$ et al.: Comparison of the pro-postoperative analgesia of intraoperative dexmedetomidine with and without loading dose following general anesthesia: a prospective, randomized, controlled clinical trial. Medicine (Baltimore) 96: e6106, 2017

Address reprint requests to: Kouhei Nii, MD, PhD, Department of Neurosurgery, Fukuoka University Chikushi Hospital, 1-1-1 Zokumyoin, Chikushino, Fukuoka 818-8502, Japan.

e-mail: k.nii@cis.fukuoka-u.ac.jp 